\title{
Effects of Sevelamer Carbonate versus Calcium Acetate on Vascular Calcification, Inflammation, and Endothelial Dysfunction in Chronic Kidney Disease
}

\author{
Darius Mason ${ }^{1}$, Kavitha Godugu ${ }^{2}$, Daryl Nnani ${ }^{3}$, and Shaker A. Mousa ${ }^{2}$ \\ ${ }^{1}$ The University of Tennessee Health Science Center \\ ${ }^{2}$ Albany College of Pharmacy and Health Sciences \\ ${ }^{3}$ Montefiore Medical Center
}

April 17, 2021

\begin{abstract}
Rationale \& Objective: Hyperphosphatemia is present in most patients with end-stage renal disease (ESRD) and has been associated with increased cardiovascular mortality. Phosphate binders (calcium-based and calcium free) are the mainstay pharmacologic treatment to lower phosphorus levels in patients with ESRD. Study Design: We evaluated biochemical markers of vascular calcification, inflammation, and endothelial dysfunction in patients with Chronic Kidney Disease (CKD) treated with sevelamer carbonate versus calcium acetate. Setting \& Participants: We enrolled 50 CKD patients (stages 3 and 4 ) and treated them with sevelamer carbonate and calcium acetate for 12 weeks. Outcomes: At the end of the study the biomarkers of vascular calcification, inflammation, and endothelial dysfunction were analyzed. Results: A significant increase in HDL-cholesterol was observed with sevelamer carbonate but not with calcium acetate. Treatment with sevelamer carbonate reduced serum phosphate, calcium phosphate, and FGF-23 levels and there was no change with calcium acetate treatment. The inflammatory markers IL$8, \mathrm{IFN}-\gamma$, and TNF $\alpha$ decreased with response to both treatments. The levels of IL- 6 significantly increased with calcium acetate treatment and no change was observed in the sevelamer carbonate treatment group. Conclusion: Sevelamer carbonate showed favorable effects on anti-inflammatory and vascular calcification biomarkers compared to calcium acetate treatment. Funding: Funding was received from Sanofi/Genzyme. Trial Registration: Registered at trial.com, registration number NCT01277497.
\end{abstract}

\section{Introduction}

Abnormalities of mineral metabolism lead to the development of vascular calcification and aortic stiffness in Chronic Kidney Disease (CKD) patients that contributes to cardiovascular disease. ${ }^{1-3}$ Despite normal values of calcium and phosphorus in the CKD population, $40 \%$ of CKD patients not receiving dialysis display evidence of calcification on imaging. ${ }^{4,5}$ Opportunities to reduce or slow the progression of vascular calcification in early stages of CKD should be explored to hinder the establishment of vascular calcification. Phosphate binders for the management of secondary hyperparathyroidism in CKD patients not receiving dialysis is mainly limited to the correction of hyperphosphatemia. Several studies have illustrated the development of inflammation and abnormal mineral metabolism prior to the development of frank hyperphosphatemia. ${ }^{6-8}$ The trade-off in the nephron hypothesis suggests that a high concentration of phosphate in the cortical distal nephron reduces the concentration of ionized calcium in that segment, and thereby necessitates increased parathyroid hormone to maintain normal calcium reabsorption and normocalcemia. ${ }^{9}$ As such, the relative normal serum phosphate provides limited value in determining the detrimental effects of phosphate exposure. ${ }^{10-12}$ In this study, the effects of sevelamer carbonate or calcium acetate were compared for their role on biomarkers indicative of vascular calcification, inflammation, and endothelial dysfunction in patients with CKD stages 3 and 4 without hyperphosphatemia. 


\section{Methods \\ Patients}

This was a randomized, prospective, open-label, parallel group study approved by both the Institutional Review Board (IRB) at the Albany College of Pharmacy and Health Sciences and the IRB at the Albany Medical Center. Fifty CKD (stages 3 and 4) patients were recruited from the Albany Medical Center South Campus facility between 2011 and 2015. Patients were included in the study if they were males or females [?] 18 years of age at the start of screening, CKD stage 3 or 4 (eGFR $15-60 \mathrm{~mL} / \mathrm{min} / 1.73 \mathrm{~m}^{2}$ ), not expected to start dialysis for 8 months, serum intact PTH $<500 \mathrm{pg} / \mathrm{mL}$ during the screening period, and a stable dose of ACEi/ARB regimen 30 days prior to screening. Over $80 \%$ of study subjects were Caucasian and male.

The duration of the study was 15 weeks in total spilt into 3 phases: screening phase, washout phase, and treatment phase (Figure 1). Written informed consent was obtained prior to any screening procedures. Study subjects' laboratory data was screened to ensure they fulfilled the inclusion criteria. The following examinations and assessments were conducted one time during the 30-day screening period: demographics, medical history, iPTH, pregnancy test (if applicable), and medication history. Study subjects were randomized by a computer program in a 1:1 ratio to receive either sevelamer carbonate or calcium acetate. If applicable, phosphate binder therapy was discontinued in study subjects receiving phosphate binder therapy 3 weeks prior to starting study medications during the washout phase. Study subjects only took phosphate binder if they were randomized to during the treatment phase. Baseline characteristics of the study subjects are presented in Table 1.

Study subjects were treated with sevelamer carbonate $1600 \mathrm{mg} 3$ times daily with meals or calcium acetate $1334 \mathrm{mg} 3$ times daily during the treatment phase for a total of 12 weeks. All patients received cholecalciferol 400 units daily during the treatment phase of the study. The treatment phase consisted of 5 total study visits: baseline study (day 1 and day 2), week 4, week 8, and week 12. The following assessments and laboratory tests were obtained on day 1: fibroblast growth factor-23 (FGF-23), serum chemistry (calcium, phosphorous albumin, alkaline phosphatase, cholesterol panel), iPTH, 25- $\mathrm{OH}, 1,25 \mathrm{OH}$, markers of calcification (fetuin-A, osteoprotegrin), inflammation (IL-6, TNF- $\alpha$, IL-10, IL-17, INF- $\gamma$ ) and vascular adhesion molecules (sICAM1, sVCAM-1, P-selectin, E-selectin). Patients were given a urine collection container and instructed to start a 24-hour urine collection. Study subjects would return on day 2 to return their 24-hour urine collection sample, which was analyzed for urinary calcium, phosphorous, protein, and creatinine. The following laboratory assessments were collected during weeks 4 and 8: FGF-23, phosphorous, calcium, and iPTH. The levels of FGF-23, serum chemistry (calcium, phosphorous albumin, alkaline phosphatase, cholesterol panel), iPTH, 25- $\mathrm{OH}, 1,25 \mathrm{OH}$, markers of calcification (fetuin-A, osteoprotegrin), inflammation (IL-6, TNF- $\alpha$, IL-10, IL17, INF- $\gamma$ ), and vascular adhesion molecules (sICAM-1, sVCAM-1, P-selectin, E-selectin) were assessed at the end of treatment phase (12 weeks).

\section{Statistics}

Continuous variables were expressed as mean \pm SD. Comparison of the biomarkers' concentrations within groups and between the two groups were performed using either student's t test or Wilcoxon rank sum test for paired differences and non-parametric data with GraphPad Prism 7 software (GraphPad, San Diego, CA) and statistical significance was defined as $P<0.05$.

\section{Results}

\section{Markers of mineral metabolism}

Changes in markers of mineral metabolism are presented in Table 2. The average serum phosphate level was within normal range for both treatment groups, $3.7 \pm 0.73$ and $3.3 \pm 0.70$ for CA and SC, respectively. In both treatments, within groups the levels of phosphate and calcium remained clinically unchanged. Sevelamer treatment resulted in a significant decrease in levels of FGF-23, calcidiol, and calcitriol, whereas FGF-23 and calcitriol remained unchanged in the calcium acetate group. A significant increase in calcidiol, was 
observed in the calcium acetate group. PTH decreased significantly in the calcium acetate group but not in the sevelamer carbonate group.

Serum lipids

Significant decreases in total cholesterol and LDL-cholesterol $(P<0.001)$ were observed with sevelamer carbonate but not with calcium acetate. Significant increases in HDL-cholesterol were observed with sevelamer carbonate but not with calcium acetate $\left(\begin{array}{ll}P & <0.05\end{array}\right)$. Triglyceride did not change from baseline in either group. No statistically significant difference was observed in triglycerides in both groups compared to baseline (Table 3).

Treatment with sevelamer carbonate significantly decreased total cholesterol, LDL, and triglycerides compared to calcium acetate (Figure 2). These changes were significantly different between groups $(* * * P<0.001$, $\left.{ }^{*} P<0.05\right)$.

\section{Inflammatory markers}

Effects of phosphate binder treatment on inflammatory markers are presented in Table 4. A significant decrease in C-reactive protein (CRP) was observed at the end of the treatment with sevelamer carbonate, whereas no change was observed in CRP in the calcium acetate group. A significant decrease was observed in IL-8, IFN- $\gamma$ and TNF- $\alpha$ with response to both treatments. The levels of IL-6 increased significantly in the calcium acetate group and no change was observed in the sevelamer carbonate group. Serum IL-10 levels decreased significantly with sevelamer carbonate treatment and no change was observed for IL-10 in the calcium acetate group.

Figure 3 shows the inflammatory profile between the calcium acetate and sevelamer groups. In patients receiving the sevelamer, serum IL-8 and IL-10 showed a significant decrease (Figure 3b and 3c). The serum TNF- $\alpha$ levels were increased in the sevelamer group (Figure 3e) and no change in IL-6, IFN- $\gamma$ levels was observed (Figure 3a and 3d).

\section{Vascular endothelial markers}

Treatment with sevelamer carbonate at week 12 resulted in a significant increase in ICAM-1, VCAM-1, Eselectin, and L-selectin. Treatment with calcium acetate showed a significant decrease in E-selctin levels and no change was observed with other endothelial markers. No statistical significance in the levels of P-selectin was observed in either treatment group (Table 5).

There were no significant differences observed in VCAM-1, E-selectin, and L-selectin levels between the groups (Figure 4b, 4d, and 4e). However, significant decreases in levels of ICAM-1 $(* * P<0.01)$ and Pselectin $\left({ }^{*} P<0.05\right)$ were observed when compared between treatment with calcium acetate and sevelamer carbonate (Figure 4a, 4c).

\section{Vascular calcification markers}

After 12 weeks of treatment with calcium acetate, there was a significant decrease in SOST, TRAcP5b, and alkaline phosphate (Table 6), whereas with sevelamer, there was an increase in alkaline phosphate levels, a decrease in SOST levels and no statistically significant decrease in TRAcP5b. No statistical changes in OPG were observed in either treatment group.

\section{Discussion}

Despite the widespread use of phosphate binders in patients with end-stage renal disease, relatively few studies have compared calcium acetate and sevelamer carbonate with respect to their biomarkers of vascular calcification, inflammation, and endothelial dysfunction in non-dialysis patients.

The beneficial effects of sevelamer carbonate on serum lipids were expected from earlier studies in CKD patients. ${ }^{13}$ Significant decrease in total cholesterol and increase in HDL-cholesterol was seen with sevelamer carbonate but not with calcium acetate. In another study performed for one year in 200 hemodialysis 
patients, sevelamer improved the progression of coronary aortic calcification compared to calcium based binders. ${ }^{14}$

In the present study, sevelamer carbonate treatment demonstrated reductions in biomarkers related to endothelial function and inflammation. As expected from earlier studies, elevated levels of calcium were more common in calcium acetate-treated patients. ${ }^{15}$ As shown in previous studies, sevelamer effects on vascular calcification might be secondary to its lower calcium load versus lipid-lowering properties. Further and consistent with earlier studies, sevelamer significantly decreased the uric acid not observed with calcium acetate. ${ }^{16}$ Johnson et al. showed that hyperuricemia is associated with insulin resistance, dyslipidemia, hypertension, and cardiovascular diseases in patients with CKD. ${ }^{17}$

In patients with CKD, FGF-23 concentrations constitutively elevate and increase progressively as kidney function worsens. ${ }^{18} \mathrm{FGF}-23$ is released in the face of phosphate overload in non-CKD and CKD patients. With reduced nephron mass, excessive FGF-23 concentrations increase phosphorous elimination per nephron maintaining normal serum phosphorous concentrations. ${ }^{19}$ This normalization is not without consequence; FGF-23 is an inhibitor of 1,25-dihydroxyvitamin D [1,25( $\mathrm{OH}) 2 \mathrm{D}]$ synthesis and further aggravates the prevalent vitamin D deficiency seen in CKD. ${ }^{20}$ Excessive levels of phosphorous and calcium are endogenous minerals capable of stimulating the phenotypic transformation of vascular smooth muscle cells into osteoblast-like cells. ${ }^{21}$ Despite normal values of calcium and phosphorous in CKD patients, $40 \%$ of CKD patients not receiving dialysis display evidence of calcification on imaging. ${ }^{22}$ In the present study, sevelamer carbonate significantly reduced the FGF-23 levels at the end of 12 weeks of study. Experimental CKD models in animals showed that reducing intestinal phosphate absorption with sevelamer $\mathrm{HCl}$ lowered serum FGF-23 and phosphorous concentrations. ${ }^{23}$ Thus FGF-23 is considered to be a logical target in early stages of CKD to slow the progression of calcification with phosphate binders. In a 6 -week period, Oliveria et al. targeted serum FGF-23 in stages 3 and 4 CKD patients with sevelamer and calcium acetate, and at 6 weeks both phosphorous binders lowered FGF-23 levels. ${ }^{24}$ However, this short-term study did not provide any evidence of reduction or progression of vascular calcification in response to FGF-23 reduction.

Furthermore, vascular calcification causes hemodynamic alterations such as reduced compliance of large conductance arteries and autonomic dysfunction. ${ }^{25}$ Stiffening of the arterial media layer as a result of calcification manifests clinically via increased elevated pulse pressure. Moreover, higher FGF-23 concentrations have been associated with increased arterial stiffness in CKD patients. ${ }^{26,} 27$ Evidence suggests these homeostatic vascular protective mechanisms are deficient or nonfunctional in CKD patients resulting in increased amounts of calcification in the CKD patient population. ${ }^{28,}{ }^{29}$ A specific biomarker of interest is osteroprotegrin. Currently, there are few published clinical trials on the effect of sevelamer treatment on levels of calcification biomarkers, specifically feutin-A in the non-dialysis population. ${ }^{30},{ }^{31}$ However, this short-term study did not provide any evidence of reduction or progression of vascular calcification in response to FGF-23 reduction.

Additionally, inflammatory cytokines have been associated with the process of vascular calcification. Studies have demonstrated that treatment with sevelamer may have anti-inflammatory effects in hemodialysis patients. ${ }^{9,} 32$ In the current study, sevelamer carbonate significantly reduced the inflammatory markers IL-6, IL-8, IL-10, CRP, TNF $\alpha$, and IFN- $\gamma$. All these inflammatory markers have been associated with adverse outcomes and complications in CKD patients. Inflammatory cytokines also activate and upregulate the expression of adhesion molecules, intercellular adhesion molecule-1 (ICAM-1), vascular cell adhesion molecule-1 (VCAM-1), E-selectin, and P-selectin, which play a fundamental role in endothelial dysfunction. Markers of endothelial function are associated with cardiovascular events. In the present study, there was a significant increase in circulating levels of ICAM-1 and VCAM-1 with sevelamer compared with calcium acetate and these results are comparable with those reported in previous studies. ${ }^{33,}{ }^{34} \mathrm{In}$ accordance with the above speculation, chronic uremia is known to be associated with elevated pro-inflammatory cytokine levels that can upregulate the expression and release of different adhesion molecules.

Systemic inflammation has been regarded as a cardiovascular risk factor. Our study demonstrated a negative correlation between serum VCAM-1 and HDL levels and a highly significant correlation between both ICAM- 
1 and VCAM-1 and CRP levels; this is the first report of such a relationship in chronic hemodialysis patients. Moreover, compared with patients with normal CRP, patients with elevated CRP had significantly increased serum ICAM-1. The above findings are particularly important because CRP is an accepted index of overall inflammatory activity and a surrogate of underlying cytokine stimulus. Based on the above results, an association between inflammation, adhesion molecule release, dyslipidemia, and atherosclerosis could be postulated.

Author contributions: D.M., D.N., and S.A.M. designed the study; K.G. carried out experiments, analyzed the data, made the figures; K.G. and S.A.M. drafted and revised the paper; all authors approved the final version of the manuscript.

Acknowledgment: This investigation would not have been possible without funding from Sanofi/Genzyme. The authors have no conflicts of interest to declare.

Disclosures: The authors have no conflicts of interest to declare.

Data Availability Statement: Research data are not shared. All data in the figures and tables are derived from the individual data measurement.

\section{References}

1. Viegas C, Araújo N, Marreiros C, Simes D: The interplay between mineral metabolism, vascular calcification and inflammation in chronic kidney disease (CKD): Challenging old concepts with new facts. Aging (Albany NY) 11: 4274-4299, 2019.

2. Lee SJ, Lee I-K, Jeon J-H: Vascular calcification-new insights into its mechanism. Int J Mol Sci 21: 2685, 2020.

3. Vahed SZ, Mostafavi S, Khatibi SMH, Shoja MM, Ardalan M: Vascular calcification: An important understanding in nephrology. Vasc Health Risk Manag 16: 167-180, 2020.

4. Affret A, Wagner S, El Fatouhi D, Dow C, Correia E, Niravong M, et al.: Validity and reproducibility of a short food frequency questionnaire among patients with chronic kidney disease. BMC Nephrol 18: 297, 2017.

5. Ketteler M, Block GA, Evenepoel P, Fukagawa M, Herzog CA, McCann L, et al.: Diagnosis, evaluation, prevention, and treatment of chronic kidney disease-mineral and bone disorder: Synopsis of the kidney disease: Improving global outcomes 2017 clinical practice guideline update. Amm Intern Med 168: 422-430, 2018.

6. Isakova T, Nickolas TL, Denburg M, Yarlagadda S, Weiner DE, Gutiérrez OM, et al.: KDOQI us commentary on the 2017 KDIGO clinical practice guideline update for the diagnosis, evaluation, prevention, and treatment of chronic kidney disease-mineral and bone disorder (CKD-MBD). Am J Kidney Dis 70: 737-751, 2017.

7. Locatelli F, Del Vecchio L, Violo L, Pontoriero G: Phosphate binders for the treatment of hyperphosphatemia in chronic kidney disease patients on dialysis: A comparison of safety profiles. Expert Opin Drug Saf 13: 551-561, 2014.

8. Hutchison AJ: Oral phosphate binders. Kidney Int 75: 906-914, 2009.

9. Ruospo M, Palmer SC, Natale P, Craig JC, Vecchio M, Elder GJ, et al.: Phosphate binders for preventing and treating chronic kidney disease-mineral and bone disorder (CKD-MBD). Cochrane Database Syst Rev 8: CD006023, 2018.

10. Chen NX, Duan D, O'Neill KD, Wolisi GO, Koczman JJ, Laclair R, et al.: The mechanisms of uremic serum-induced expression of bone matrix proteins in bovine vascular smooth muscle cells. Kidney Int 70: 1046-1053, 2006. 
11. Muteliefu G, Enomoto A, Jiang P, Takahashi M, Niwa T: Indoxyl sulphate induces oxidative stress and the expression of osteoblast-specific proteins in vascular smooth muscle cells. Nephrol Dial Transplant 24: 2051-2058, 2009.

12. Zheng CM, Lu KC, Wu CC, Hsu YH, Lin YF: Association of serum phosphate and related factors in esrd-related vascular calcification.Int J Nephrol 2011: 939613, 2011.

13. Vlassara H, Uribarri J, Cai W, Goodman S, Pyzik R, Post J, et al.: Effects of sevelamer on HbA1c, inflammation, and advanced glycation end products in diabetic kidney disease. Clin J Am Soc Nephrol 7: 934-942, 2012.

14. Chertow GM, Burke SK, Raggi P, Treat to Goal Working G: Sevelamer attenuates the progression of coronary and aortic calcification in hemodialysis patients. Kidney Int 62: 245-252, 2002.

15. Qunibi WY, Hootkins RE, McDowell LL, Meyer MS, Simon M, Garza RO, et al.: Treatment of hyperphosphatemia in hemodialysis patients: The Calcium Acetate Renagel Evaluation (CARE Study). Kidney Int 65: 1914-1926, 2004.

16. Evenepoel P, Selgas R, Caputo F, Foggensteiner L, Heaf JG, Ortiz A, et al.: Efficacy and safety of sevelamer hydrochloride and calcium acetate in patients on peritoneal dialysis. Nephrol Dial Transplant 24: 278-285, 2009.

17. Johnson RJ, Kivlighn SD, Kim YG, Suga S, Fogo AB: Reappraisal of the pathogenesis and consequences of hyperuricemia in hypertension, cardiovascular disease, and renal disease. Am J Kidney Dis 33: 225-234, 1999.

18. Fliser D, Kollerits B, Neyer U, Ankerst DP, Lhotta K, Lingenhel A, et al.: Fibroblast growth factor 23 (FGF23) predicts progression of chronic kidney disease: The mild to moderate kidney disease (MMKD) study. J Am Soc Nephrol 18: 2600-2608, 2007.

19. Gutiérrez OM, Januzzi JL, Isakova T, Laliberte K, Smith K, Collerone G, et al.: Fibroblast growth factor-23 and left ventricular hypertrophy in chronic kidney disease. Circulation 119: 2545, 2009.

20. Gutierrez O, Isakova T, Rhee E, Shah A, Holmes J, Collerone G, et al.: Fibroblast growth factor23 mitigates hyperphosphatemia but accentuates calcitriol deficiency in chronic kidney disease. $J$ Am Soc Nephrol 16: 2205-2215, 2005.

21. Giachelli CM, Jono S, Shioi A, Nishizawa Y, Mori K, Morii H: Vascular calcification and inorganic phosphate. Am J Kidney Dis38: S34-37, 2001.

22. Russo D, Palmiero G, De Blasio AP, Balletta MM, Andreucci VE: Coronary artery calcification in patients with CRF not undergoing dialysis. Am J Kidey Dis 44: 1024-1030, 2004.

23. Nagano N, Miyata S, Abe M, Kobayashi N, Wakita S, Yamashita T, et al.: Effect of manipulating serum phosphorus with phosphate binder on circulating pth and FGF23 in renal failure rats. Kidney Int 69: 531-537, 2006.

24. Oliveira RB, Cancela AL, Graciolli FG, Dos Reis LM, Draibe SA, Cuppari L, et al.: Early control of pth and FGF23 in normophosphatemic CKD patients: A new target in CKD-MBD therapy? Clin J Am Soc Nephrol 5: 286-291, 2010.

25. Sigrist M, Bungay P, Taal MW, McIntyre CW: Vascular calcification and cardiovascular function in chronic kidney disease. Nephrol Dial Transplant 21: 707-714, 2006.

26. Jean G, Terrat JC, Vanel T, Hurot JM, Lorriaux C, Mayor B, et al.: High levels of serum fibroblast growth factor (FGF)-23 are associated with increased mortality in long haemodialysis patients. Nephrol Dial Transplant 24: 2792-2796, 2009. 
27. Llauradó G, Megia A, Cano A, Giménez-Palop O, Simón I, González-Sastre M, et al.: FGF-23/vitamin D axis in type 1 diabetes: The potential role of mineral metabolism in arterial stiffness. PloS One 10: e0140222, 2015 .

28. Moe SM, Reslerova M, Ketteler M, O'Neill K, Duan D, Koczman J, et al.: Role of calcification inhibitors in the pathogenesis of vascular calcification in chronic kidney disease (CKD). Kidney Int 67: 2295-2304, 2005 .

29. Brown RB, Razzaque MS: Dysregulation of phosphate metabolism and conditions associated with phosphate toxicity. Bonekey Rep 4: 705, 2015.

30. Tousoulis D, Siasos G, Maniatis K, Oikonomou E, Vlasis K, Papavassiliou AG, et al.: Novel biomarkers assessing the calcium deposition in coronary artery disease. Curr Med Chem 19: 901-920, 2012.

31. D'Marco L, Bellasi A, Raggi P: Cardiovascular biomarkers in chronic kidney disease: State of current research and clinical applicability.Dis Markers 2015: 586569, 2015.

32. Wang C, Liu X, Zhou Y, Li S, Chen Y, Wang Y, et al.: New conclusions regarding comparison of sevelamer and calcium-based phosphate binders in coronary-artery calcification for dialysis patients: A meta-analysis of randomized controlled trials. PloS One 10: e0133938, 2015.

33. Arici M, Kahraman S, Gençtoy G, Altun B, Kalyoncu U, Oto A, et al.: Association of mineral metabolism with an increase in cellular adhesion molecules: Another link to cardiovascular risk in maintenance haemodialysis? Nephrology Dialysis Transplantation 21: 999-1005, 2006.

34. Papayianni A, Alexopoulos E, Giamalis P, Gionanlis L, Belechri AM, Koukoudis P, et al.: Circulating levels of ICAM-1, VCAM-1, and MCP-1 are increased in haemodialysis patients: Association with inflammation, dyslipidaemia, and vascular events. Nephrol Dial Transplant 17: 435-441, 2002.

\section{Figure Legends}

Figure 1: The flow chart of study design and procedure. SA, sevelamer carbonate; CA: calcium acetate.

Figure 2: Changes in serum lipids after 12 weeks of treatment with sevelamer carbonate and calcium acetate. a) TC, total cholesterol, b) HDL-C, high-density-lipoprotein cholesterol, c) TG, triglycerides, d) LDL-C, low-density lipoprotein cholesterol. Mean $\pm \mathrm{SD}{ }^{*} P<0.05,{ }^{* * *} P<0.001$.

Figure 3: Changes in serum inflammatory markers after 12 weeks of treatment with sevelamer carbonate and calcium acetate. a) Interleukin-6, b) Interleukin-8, c) Interleukin-10, d) IFN- $\gamma$, e) TNF- $\alpha$. Values expressed as mean $\pm \mathrm{SD} * * P<0.01, * * * P<0.001$.

Figure 4: a) ICAM-1, b) VCAM-1, c) P-selectin, d) E-selectin, e) L-selectin. Values expressed as mean \pm $\mathrm{SD} * P<0.05,{ }^{* *} P<0.01$.

\section{Hosted file}

Figures1-4.pdf available at https://authorea.com/users/408322/articles/518378-effects-ofsevelamer-carbonate-versus-calcium-acetate-on-vascular-calcification-inflammation-andendothelial-dysfunction-in-chronic-kidney-disease

\section{Hosted file}

Tables.pdf available at https://authorea.com/users/408322/articles/518378-effects-ofsevelamer-carbonate-versus-calcium-acetate-on-vascular-calcification-inflammation-andendothelial-dysfunction-in-chronic-kidney-disease 